\title{
LA FORMACIÓN INICIAL DE HABILIDADES PROFESIONALES DEL INGENIERO INDUSTRIAL DESDE EL CONTEXTO DE LA MATEMÁTICA
}

The Initial Formation of Professional Skills for Industrial Engineers from the Mathematics Context

\section{Domingo Curbeira Hernández María de Lourdes Bravo Estévez Gisela Bravo López}

Resumen: Uno de los aspectos más importantes que debe ser considerado en el proceso de formación profesional inicial de las diferentes carreras de ingeniería es el relacionado con la formación y desarrollo de habilidades. El establecimiento de vínculos entre el tratamiento de los contenidos y las habilidades profesionales es tema de interés de los profesores. La formación inicial de habilidades profesionales tiene un espacio en la preparación de los Ingenieros Industriales y debe realizarse desde la impartición de las diferentes asignaturas del currículo que son tratadas en el proceso de enseñanza aprendizaje. En este trabajo se realiza un análisis de los criterios de diferentes autores en torno al tema objeto de análisis; se ofrece además un concepto de habilidad profesional y se establece un paralelo entre el proceso de formación de conceptos matemáticos y el proceso de formación inicial de una habilidad profesional de este ingeniero.

Palabras claves: Habilidad profesional, acciones, operaciones, tratamiento de conceptos matemáticos. 
Domingo Curbeira Hernánder, María de Lourdes Bravo Estévez, Gisela Bravo Lóper.

Abstract: Among the most important aspects to be considered in the initial training process in the different careers of engineering, are found what is related to the training and skills development. The linkage between the treatment of contents that students receive and professional skills is a topic of interest to teachers. Initial training of professional skills has a significant place in the preparation of Industrial Engineers and must be carried out from the teaching of the different subjects of the curriculum. This paper is an analysis of the criteria of different authors on the subject under analysis, provides a concept of professional skill and draws a parallel between the formation of mathematical concepts and the initial training of a skill in this engineer.

Keywords: professional skill, actions, operations, formation of mathematical concepts.

\section{Introducción}

El problema de la formación general de un profesional, en especial, en lo que concierne al proceso de formación inicial de habilidades profesionales es uno de los más actuales en las ciencias pedagógicas y en la práctica educativa en los últimos tiempos. Es por ello que numerosos pedagogos, psicólogos, sociólogos, filósofos, entre otros, han dedicado especial atención a este aspecto. Importantes contribuciones pueden hacerse desde la impartición de las asignaturas del ciclo de formación básica al proceso de formación inicial de habilidades profesionales, en el caso que ocupa se establece un vínculo entre el tratamiento de los conceptos del cálculo integral en la disciplina Matemática General y la habilidad profesional diseñar soluciones y visionar estrategias con rigor científico, 
declarada en el Modelo del Profesional del Ingeniero Industrial, entendiéndose por visionar dar una opinión profesional sobre una problemática cualquiera.

Desde el comienzo mismo de la enseñanza de las ingenierías se trabaja por lograr un profesional, cuya preparación esté acorde con el desarrollo de la época en que se desenvuelve y en las que este vive, así como también la manera como debe enfrentarse la formación de dichos profesionales. Los retos que asume la Educación Superior del presente siglo, están dirigidos a lograr que los futuros profesionales que se preparan encuentren en el proceso de formación profesional inicial los mecanismos mediante los cuales se eleven los motivos por la profesión que han elegido, permitiendo así un alto compromiso, implicación e interés por las diferentes actividades propias de la formación profesional inicial.

\section{La enseñanza de la Matemática en la formación profesional inicial del Ingeniero Industrial}

En opinión de Dujet (2007), los ingenieros están destinados a evolucionar en un mundo de complejidad creciente y cada vez más incierto, donde las nuevas habilidades que se requieren descansan necesariamente en el aprendizaje de las herramientas y en el conocimiento de las heurísticas (arte de inventar o descubrir) de las teorías matemáticas que les dieron vida. El ingeniero debe poder elegir, con conocimiento de causa, el modelo matemático que mejor se adapte al nivel de complejidad con el que se va a enfrentar y así determinar los parámetros concurrentes y sus ajustes según el problema estudiado, tomando en cuenta, cualquiera que sea su naturaleza, las incertidumbres relacionadas con el contexto y ser capaz de justificar la gestión y el tratamiento de dichas incertidumbres.

La disciplina Matemática General, junto a otras, posibilita que se desarrollen los fundamentos de la formación de un especialista en 
Domingo Curbeira Hernánder, María de Lourdes Bravo Estévez, Gisela Bravo Lóper.

Ingeniería Industrial, ya que todo ingeniero considera representaciones técnicas y científicas en términos puramente matemáticos con los cuales puede trabajar los rasgos cuantitativos de los fenómenos que estudia. El objetivo de esta disciplina es lograr que el ingeniero industrial domine el aparato matemático que lo haga capaz de modelar y analizar los procesos técnicos, económicos, productivos y científicos, empleando para ello procedimientos analíticos y/o numéricos, haciendo un uso eficiente de las diferentes técnicas de cómputo, desarrollando así su pensamiento lógico, heurístico y algorítmico (Colectivo de autores, 2007).

El estudio de las matemáticas realiza importantes contribuciones a la formación del Ingeniero Industrial, pues amplía la madurez matemática y la capacidad de trabajo con la abstracción, desarrolla habilidades para la comunicación de propiedades y características de magnitudes en forma gráfica, numérica, simbólica y verbal. Además, contribuye a conformar una cultura científica general actualizada que tiene en cuenta aspectos tales como: el uso de la informatización en la resolución de problemas, el procesamiento de literatura técnica, así como el manejo adecuado del lenguaje interdisciplinario.

Se destaca que de las 832 que reciben los estudiantes, del primer año de Ingeniería Industrial, correspondientes al currículo base, el $30.76 \%$ se dedica a la enseñanza de la Matemática, es decir, 256 horas son distribuidas en tres asignaturas de esta disciplina, Matemática I (96), Álgebra Lineal y Geometría Analítica (con 64 horas) y Matemática II (con 96 horas).

Señala Dujet (2007), que los programas de matemáticas para ingenieros deben tener como objetivos primarios, entre otros, los siguientes:

- Las matemáticas, "lenguaje de todas las ciencias" son necesarias para que los estudiantes puedan llegar a comprender las otras ciencias, así como para ayudarle a adquirir las técnicas y los 
La formación inicial de habilidades profesionales del ingeniero industrial desde el contexto de la matemática

métodos que constituyen las herramientas que le son imprescindibles.

- Aprender a reconocer los presupuestos del sistema en estudio y comprender que toda ciencia no es más que una primera aproximación.

- Saber manejar los programas informáticos cada vez más sofisticados, para lograr un buen conocimiento de las heurísticas subyacentes con la finalidad de poder adaptarse al contexto, aspecto que también forma parte del arte del ingeniero.

- Desarrollar "el espíritu de geometría y de sutileza", es decir, saber identificar lo que se presenta como evidencia (tangible y contingente), procurando tener al mismo tiempo la apertura de espíritu y la flexibilidad que permiten ir más allá de las apariencias (en el análisis de un problema...). Esta dualidad eminentemente "desarrollable" por las matemáticas tomará su eficiencia en la transferencia a la tecnología del ingeniero.

- El programa analítico de la disciplina Matemática General para el Ingeniero Industrial de las universidades cubanas considera como objeto de la matemática todas las formas y relaciones del mundo real que posean objetivamente tal grado de independencia respecto al contenido, que pueden ser totalmente abstraídas de este último. Además, no solo las formas abstraídas de la realidad son objeto de estudio de la matemática sino también aquellas lógicamente posibles, determinadas sobre la base de formas y relaciones ya conocidas (Colectivo de Autores, 2007).

La importancia esencial de la Matemática en la formación del Ingeniero Industrial radica en ser el lenguaje de la modelación, el soporte simbólico con la ayuda del cual se expresan las leyes que gobiernan el objeto de trabajo de este ingeniero, otorgándose por ello prioridad al desarrollo de la capacidad de modelar utilizando los conceptos y el lenguaje de la Matemática, así como la habilidad 
Domingo Curbeira Hernánder, María de Lourdes Bravo Estévez, Gisela Bravo Lóper.

de interpretar modelos ya creados sobre la base de los conceptos de la disciplina.

Los autores de este trabajo, coincidiendo con Escalona (2011), entienden que la enseñanza de la matemática tiene que estar contextualizada asumiéndose en este caso la posición teórica del investigador Ortigoza (2006) cuando expresa: "El aprendizaje contextual tiene lugar cuando el alumno procesa la información y los conocimientos nuevos de tal manera que le da sentido en su marco de referencia (su propio mundo interno de memoria, experiencia y respuesta)". Para este autor, la contextualización del aprendizaje se puede lograr aplicando las siguientes estrategias:

- Relación: proceso de aprendizaje realizado en el contexto de la experiencia de la vida.

- Experimentación: aprender a partir de la exploración, descubrimiento e invención.

- Aplicación: emplear el contenido y la información en un contexto útil.

- Cooperación: lograr compartir, interactuar y comunicarse.

- Transferencia: consiste en aprender usando el conocimiento que ya tiene el alumno en un nuevo contexto o una nueva situación; es decir, se construye por encima de lo que el alumno ya sabe.

Es de señalar que las estrategias no pueden cumplirse totalmente en el marco de una clase, por lo que solo se presentan algunos rasgos de ellas, especialmente las de experimentación, aplicación y transferencia. Aquí lo importante es establecer las relaciones del contenido con los diferentes contextos de actuación de los futuros profesionales (por ejemplo, la formación inicial de habilidades profesionales a través del tratamiento de conceptos matemáticos, aspecto que se concretará en el marco de las clases a corto y largo 
La formación inicial de habilidades profesionales del ingeniero industrial desde el contexto de la matemática

plazos respectivamente), principalmente a través de la creación y resolución de modelos relativos a estas áreas de la ciencia.

\section{La formación y el desarrollo de habilidades}

En el trabajo con las habilidades se distinguen dos etapas: la de formación y la de desarrollo; la primera comprende la adquisición consciente de los modos de actuar, cuando bajo la dirección del profesor, el estudiante recibe la orientación adecuada sobre la forma de proceder y una vez adquiridas estas habilidades, se inicia el proceso de ejercitación que comprende la segunda etapa, determinándose el nivel de desarrollo por la rapidez y corrección con que la acción se ejecute.

El problema relacionado con el proceso de formación y desarrollo de habilidades ha sido ampliamente estudiado e investigado, Mulet (2006) señala a los autores: Ananiev, Bodaliev, Galperin, Leontiev, Petrovsky, Rubinstein, Lomov, Ponomariev, los que centraron su atención en la teoría de la actividad. En la teoría de la formación de las acciones mentales por etapas, se mencionan a: Galperin, Talizina, Elkonin; mientras que en la teoría sobre habilidades y hábitos, lo han hecho los autores: Kabalova-Meller, Menchinskaia, Dmitriv; por otra parte en la esencia y estructura de las actividades pedagógicas se citan los autores: Kuzmina, Slationi y a Sherbakov.

Refiriéndose al caso concreto de Cuba, Mulet (2006) cita a Brito, Rey, Hurtado, Maura: autores que han estudiado los aspectos relacionados con la formación y desarrollo de las habilidades generales. Un aporte teórico en este campo lo realizó, en los últimos tiempos Álvarez, quien ha tenido como seguidores a González, Ramírez, Martínez y Gómez (Mulet, 2006).

Petrovsky (1980) asume la habilidad como el dominio de un complejo sistema de acciones psíquicas y prácticas necesarias para una regulación racional de la habilidad, con ayuda de conocimientos y hábitos que la persona posee. Las habilidades constituyen la 
Domingo Curbeira Hernández, María de Lourdes Bravo Estéver, Gisela Bravo Lóper.

relación del individuo con el objeto y su elemento rector es el motivo, o sea, la necesidad de realizar algo. Como plantea Brito (1987), la habilidad es el dominio de las técnicas de la actividad, tanto cognoscitivas como prácticas. Talizina (1988) asume que en el proceso de asimilación de la habilidad se debe partir de una imagen de las acciones a realizar, por lo que plantea como componente de la habilidad a su imagen generalizada y considera que esto forma parte del proceso de aprendizaje y no de la estructura de la habilidad en sí.

La habilidad es asumida por Bermúdez y Rodríguez (1996) como formación psicológica, que tiene su fundamento en la estructura de la personalidad y particularmente en la esfera de autorregulación ejecutora (cognitivo instrumental), en la que coexisten las unidades psíquicas: estado cognitivo, estado metacognitivo e instrumentación ejecutora, de la que es tributaria junto a otras manifestaciones de las ejecuciones: acciones, operaciones, hábitos y capacidades.

La idea general que transmiten las definiciones anteriores, además de centrarse en el campo psicológico es que las habilidades por sí solas no determinan el éxito en la resolución de una tarea, sino que es necesario tener presente los conocimientos y hábitos que el sujeto posee.

Se asume en este trabajo la definición aportada por Álvarez (1999) que, coincidiendo con otros autores, considera desde el punto de vista psicológico la habilidad como un sistema de acciones y operaciones para elaborar la información contenida en los conocimientos y que lleva al logro de un propósito determinado. Además, la describe desde el ámbito pedagógico y valora al individuo como un ente social, activo y desarrollador de la cultura. La describe como la dimensión del contenido que muestra el comportamiento de la persona en una rama del saber propio de la cultura de la humanidad. 
La autora Bravo (2002), refiriéndose a la formación de las habilidades justifica que las mismas constituyen uno de los objetivos fundamentales del proceso docente-educativo, siendo estas las que permiten a la persona poder realizar una determinada tarea, dependiendo del éxito de las destrezas que se tengan al respecto. Estas forman parte del contenido de una asignatura, caracterizan las acciones que el estudiante realiza al interactuar con el objeto de estudio y su desarrollo permite la asimilación del conocimiento.

Montes de Oca (2002), refiriéndose al concepto de habilidad propiamente dicho, considera que se emplea con frecuencia en la literatura psicológica y pedagógica actual, pero su estudio constituye aún un problema abierto y amplio para la ciencia pues se aprecian lógicas divergencias e incluso discrepancias científicas en los puntos de vista de los autores, debido a que no todos definen el concepto en términos similares, no todos coinciden plenamente sobre cuáles deben ser sus componentes, los requisitos y condiciones fundamentales a tener en cuenta para su formación y desarrollo. Además señala que el significado de términos tales como: aptitud, capacidad, habilidad, destreza y competencia, suelen originar ocasionalmente un problema que podría llamarse de circularidad, pues algunos de los términos mencionados son definidos recurriendo a los otros y es difícil lograr establecer una clara diferenciación entre ellos, o explicar la forma en que se vinculan y/o complementan.

Los autores de este trabajo coinciden con Mulet (2006), quien considera que el problema sobre la naturaleza psicológica y pedagógica de las habilidades aún no ha tenido una solución única, evidenciándose en los diferentes criterios que existen en la actualidad.

La actividad no es solamente la vía por la que se puede determinar la existencia de una habilidad, sino también la condición de su perfeccionamiento, de ahí que el profesor, al dirigir el proceso de formación y desarrollo de las habilidades, debe estructurar de manera adecuada la actividad de sus estudiantes teniendo en cuenta tanto las 
Domingo Curbeira Hernánder, María de Lourdes Bravo Estévez, Gisela Bravo Lóper.

condiciones psicopedagógicas generales como las específicas de su asignatura. Criterio con el que este autor coincide perfectamente.

Al comparar el concepto de habilidad con el de actividad, los autores Izquierdo y Corona (2012) aportan elementos con los que se coincide, al observar que se presentan aspectos valorativos que enriquecen la percepción didáctica y que contribuyen a entender, desde el punto de vista epistemológico, lógico y metodológico, la necesidad de investigar en el tema que ocupa la atención de este investigador también. Los autores de este trabajo ilustran dicha relación mediante la siguiente tabla.

\begin{tabular}{|c|l|l|l|}
\hline \multicolumn{2}{|c|}{ Estructura de la actividad } & \multicolumn{2}{c|}{ Estructura de la habilidad } \\
\hline $\begin{array}{c}\text { Componentes } \\
\text { ejecutores }\end{array}$ & \multicolumn{1}{|c|}{$\begin{array}{c}\text { Componentes } \\
\text { inductores }\end{array}$} & $\begin{array}{c}\text { Componentes } \\
\text { ejecutores }\end{array}$ & $\begin{array}{c}\text { Componentes } \\
\text { inductores }\end{array}$ \\
\hline Actividad & Objetivo principal & & Objetivos - Motivos \\
\hline Acciones & Objetivos subordinados & Acciones & \\
\hline Operaciones & $\begin{array}{l}\text { No tienen objetivos, } \\
\text { dependen de las } \\
\text { condiciones y de los } \\
\text { sujetos o de ambos }\end{array}$ & Operaciones & \\
\hline
\end{tabular}

En la tabla anterior se observa un elemento común que determina el nexo entre actividad y habilidad, es que ambas se ejecutan mediante un sistema de acciones y operaciones, las que a su vez se relacionan con el objetivo de la actividad según se muestra en los mismos.

Como cita Cañedo (2004) toda acción se descompone en varias operaciones, con determinada lógica, donde las operaciones son pequeñas acciones, son procedimientos, son las formas de realización de la acción atendiendo a las condiciones, es decir a las circunstancias reales en las cuales se realiza la habilidad, en fin, le dan a la acción esa forma de proceso continuo. Más adelante, el autor plantea que tanto el concepto de acción como de operación 
La formación inicial de habilidades profesionales del ingeniero industrial desde el contexto de la matemática

son relativos pues en la etapa de formación de una habilidad lo que interviene como acción, puede en un momento dado convertirse en operación y viceversa.

En este artículo se aborda el proceso de formación de una habilidad profesional en estudiantes de Ingeniería Industrial, más adelante se presentan las acciones y operaciones que responden a dicha formación, tal y como ha quedado explicitado en la definición aportada por Álvarez (1999), el sujeto una vez que domina estas acciones y operaciones puede interactuar con el objeto para transformarlo, conocerlo, humanizarlo como plantea el propio autor.

A través del desarrollo de las habilidades específicas de una asignatura se logra el desarrollo de las habilidades generales, pero también se realizan importantes contribuciones al proceso de formación inicial de las habilidades profesionales.

\section{Las habilidades profesionales}

El proceso de formación inicial de habilidades profesionales constituye un caso particular a tener en cuenta entre el conjunto de habilidades, son tratadas en el proceso docente educativo, estas son las propias del ejercicio de la profesión y se definen en función de la asimilación por el estudiante de los modos de actuación de aquella actividad que está relacionada con el campo de acción de su futura labor y que tiene como base los conocimientos de la carrera, los hábitos inherentes a su profesión y los valores que deben formarse.

Una definición de este tipo particular de habilidad es ofrecida por Miari (1982) el que plantea que habilidad profesional es "la disposición a efectuar la acción o el conjunto de acciones productivas de una manera consciente, utilizando correctamente, en situaciones dadas, los métodos oportunos de su realización, logrando adecuados resultados cualitativos y cuantitativos en el trabajo" (Miari, 1982: 242). 
Domingo Curbeira Hernánder, María de Lourdes Bravo Estévez, Gisela Bravo Lóper.

Se destaca en este caso no solo la disposición hacia la acción física que debe realizarse en el proceso productivo, sino también hacia lo intelectual, a partir de la disposición a realizar tareas de un determinado puesto de trabajo, se trata de encontrar la forma más efectiva para resolverlas, teniendo en cuenta el ahorro de recursos y el cuidado del entorno laboral, planificarlas anticipadamente y ejecutarlas con exactitud.

En opinión de Márquez (1993) [las habilidades] "son aquellas que garantizan el éxito en la ejecución de la actividad de la profesión y la solución de los más diversos problemas de esa especialidad.” (Márquez, 1993:100). En esta definición se destaca la importancia de las habilidades profesionales para lograr un buen desarrollo de las tareas de los puestos de trabajo, así como la solución de los problemas que se presenten, por lo que se deduce que son aquellas que tienen como base los problemas profesionales de una especialidad determinada, y que son reflejadas en el perfil ocupacional o en el modelo del profesional.

Mestre (1995) las define como: "Habilidades previstas en el contenido del proceso docente educativo que se corresponden con los modos de actuación del profesional dado y han de tener un nivel de sistematización tal que, una vez apropiado de ellas, le será posible al estudiante enfrentar y dar solución a múltiples problemas profesionales" (Mestre, 1995: 22). A diferencia de los autores anteriores se observa que el autor las concibe formando parte del contenido del proceso docente educativo, proceso a partir del cual debe potenciarse su formación, para luego ser aplicadas en actividades puramente profesionales.

Para Álvarez (1996), "habilidad profesional es aquella que permite al egresado integrar los conocimientos y elevarlos al nivel de aplicación profesional, dominar la técnica para mantener la información actualizada, investigar, saber establecer vínculos con el contexto social y gerenciar recursos humanos y materiales" (Álvarez, 1996: 27). En esta definición se distingue primero una 
La formación inicial de habilidades profesionales del ingeniero industrial desde el contexto de la matemática

etapa de formación junto con la adquisición de conocimiento para luego poder aplicarlas a la solución de diversos problemas en el contexto de la práctica.

Para Fuentes (1999) constituyen "el contenido lógico de las acciones que realiza el profesional al interactuar con los objetos de la profesión" (Fuentes, 1999: 35). Se señala en este caso que se conciben las acciones que el profesional debe aplicar para solucionar problemas propios de su profesión.

En opinión de Rodríguez (2012), las habilidades profesionales constituyen una dimensión del contenido como componente del proceso de enseñanza aprendizaje y se manifiestan en el sistema de acciones intelectuales y prácticas que al sistematizarse y ejercitarse permiten el desempeño integral en la realización de labores productivas y en la solución de problemas de carácter general inherentes a las tareas y ocupaciones expresadas en su calificador de cargo. El autor las enmarca como dimensión del contenido y refiere como esencial las acciones que debe ejecutar el profesional para la solución de los problemas propios de la profesión.

Como se observa todos los autores que se mencionan son concurrentes al considerar que en el caso de las habilidades profesionales, estas se corresponden con los modos de actuación del profesional, y por tanto no se enmarcan las habilidades profesionales desde las diferentes asignaturas del currículo, aunque algunos destacan la importancia de su formación a través del proceso docente educativo.

Del estudio realizado sobre las definiciones descritas, se aprecia que se hace reconocimiento a la significación de la experiencia histórica asimilada por el hombre para la ejecución de las acciones psíquicas y prácticas con un carácter consciente, se declara que las operaciones despliegan a las acciones con arreglo a las condiciones de realización de la tarea y se expresa que estas acciones generan el aprendizaje de modos de actuación en la personalidad. A partir de 
Domingo Curbeira Hernánder, María de Lourdes Bravo Estévez, Gisela Bravo Lóper.

las definiciones anteriores los autores de este trabajo definen a las habilidades profesionales como:

... acciones y operaciones, subordinadas a una profesión concreta, que pueden formar parte de una asignatura o de varias asignaturas ya sea de forma implícita o explícita; que son sistematizadas en el proceso de la actividad, responden a un objetivo concreto y permiten la solución de los más diversos problemas de una determinada profesión.

Para la elaboración de la definición anterior se tuvo en cuenta que:

- Una acción constituye un sistema complejo de operaciones, de forma sistemática y consciente necesarias para la regulación de la actividad.

- Las acciones se relacionan con los objetivos, las operaciones con las condiciones.

- Toda acción incluye un determinado conjunto de operaciones, que son ejecutadas según un orden determinado.

Es importante, como bien señala Llanes (2010), que el estudiante conozca las acciones y operaciones de la habilidad que realizará, para que asuma la tarea con responsabilidad y esté consciente del resultado que tiene que alcanzar, así como del procedimiento que seguirá hasta lograrlo, de modo que se sienta implicado, seguro y motivado con el trabajo, y sea capaz de realizar un autocontrol sobre cada paso que ejecuta y de autovalorarse en el desempeño de su rol profesional.

Las sociedades del siglo XXI precisan de una actividad transformadora sustentada sobre sólidos cimientos científicos, que se basan en la situación económica y social, con un enfoque histórico, que constituyen criterios básicos a tener en cuenta.

El proceso de formación de las habilidades profesionales es concebido en esta investigación desde la formación básica de este 
futuro profesional, es decir, a través del trabajo socializador que se desarrolla desde la clase, se forma a un individuo que actúa de forma consciente, adoptando así una posición activa ante la vida y una actitud enfocada hacia el deber social.

Se destaca, además, la importancia que se le atribuye al tratamiento de conceptos matemáticos para a partir de ellos potenciar el proceso de formación inicial de habilidades profesionales. Concebir el proceso de formación de habilidades profesionales desde la formación básica, significa en este caso hacer un paralelo entre el proceso de formación de conceptos matemáticos, en particular, los conceptos del cálculo integral y el proceso de formación inicial de habilidades profesionales. Abarcar la contribución desde una disciplina a la formación inicial de las habilidades profesionales es una tarea extremadamente compleja, por ello se restringe al caso de la formación de la habilidad profesional diseñar soluciones y visionar estrategias con rigor científico desde la disciplina Matemática General en el primer año de Ingeniería Industrial.

\section{Acciones y operaciones para el tratamiento de los conceptos del cálculo integral}

Las acciones y operaciones que se presentan para el caso del tratamiento de conceptos deben ejecutarse siempre que se trabaje en la elaboración de un concepto del cálculo integral, estas acciones y operaciones no son una camisa de fuerza, su aplicación no tiene por qué ser rígida, los estudiantes tienen la posibilidad de ser creativos y para ello pueden formularse preguntas, las que ayudarán a razonar la efectividad de su aplicación.

Las acciones y operaciones deben ser aprendidas e interiorizadas, reflexionando sobre ellas y pasando por una serie de éxitos y fracasos, ante estas últimas es conveniente revelar las causas que llevaron a las dificultades y comprenderlas como tal, para que tenga sentido el por qué un razonamiento es correcto para un problema 
Domingo Curbeira Hernánder, María de Lourdes Bravo Estévez, Gisela Bravo Lóper.

y no para otro. Estas deben ser elaboradas cooperativamente con los estudiantes, mediante una conversación heurística, donde a partir de la reflexión con los mismos debe analizarse cuál es el paso necesario a dar. Al concluir el proceso de formación del concepto el profesor debe hacer un análisis para determinar todas las acciones y operaciones que forman parte del procedimiento.

Las acciones y operaciones que proponen los autores para el tratamiento de un concepto del cálculo integral se muestran en lo adelante:

- Reactivar los medios que tienen implicación en el nuevo concepto a definir, recopilando toda la información, independientemente de su utilidad o no.

En esta acción los estudiantes guiados por el profesor deben buscar los medios (teoremas, definiciones, conceptos y otras propiedades que guardan relación con el concepto a tratar), independientemente de que resulten de utilidad o no, el profesor apoya con preguntas para que los estudiantes puedan determinar qué elementos del conocimiento tienen relación directa o indirecta con la problemática que se propuso o se propone.

- Modelar geométricamente el problema planteado, si es posible.

En este caso el profesor reflexiona con los estudiantes en relación con el problema geométrico presentado, se determina si se trata de un problema geométrico del plano o del espacio; si se trata de un problema del plano, entonces habrá que determinar las curvas planas que limitan o conforman a la región, por lo que se deberá esbozar dicha región. En el caso de un problema del espacio se procede de forma similar, es decir, se determinará cuáles son las superficies que conforman el sólido y a continuación deberá esbozarse el mismo.

- Reducir el problema planteado a un problema resuelto con anterioridad. 
Si el problema presentado es del plano, entonces habrá que realizar una partición de dicha región, a continuación dividirla en regiones equivalentes para posteriormente realizar los cálculos pertinentes. Si se trata de una región del espacio, habrá que realizar una proyección de dicha región en uno de los planos proyectantes, luego realizar una partición de la región y posteriormente dividirla en sólidos equivalentes para realizar los cálculos pertinentes.

- Realizar la construcción de los objetos correspondientes.

Es el momento de construir el objeto (integral definida, doble, triple, curvilínea o de superficie) deseado a partir de las consideraciones realizadas; es importante observar que el objeto abstracto construido se obtiene a partir de un objeto anteriormente elaborado (integral definida). La esencia de esta acción es finalmente comprender el objeto abstracto construido.

- Explicar y/o formular una definición del concepto sobre la base de los resultados alcanzados.

Aquí, a partir de los elementos obtenidos, se da una explicación de los resultados obtenidos para a partir de ellos poder explicar o formular una definición del concepto tratado.

- Realizar consideraciones retrospectivas y perspectivas.

Es una de las acciones más importantes, pues es el momento de incorporar el concepto tratado en el sistema de conceptos y por tanto en el sistema de conocimiento de los estudiantes, se analiza si es necesario realizar el tratamiento de algún caso especial o límite (aplicaciones del objeto construido al cálculo), si la vía que se ha seguido puede ser utilizada en casos similares o posteriores.

En estas acciones y operaciones los estudiantes no tienen por qué memorizarlas, una vez que son aprehendidas, estos pueden actuar en correspondencia con ello, es decir, deben entenderlas y 
Domingo Curbeira Hernández, María de Lourdes Bravo Estéver, Gisela Bravo Lóper.

comprenderlas. Como ya fue reiterado, estas acciones deben ser elaboradas junto con los estudiantes como copartícipes de la elaboración, para que logren su comprensión y desarrollen la habilidad.

Una vez concluido este tratamiento se proponen a los estudiantes las acciones y operaciones de la habilidad profesional que comienza a formarse, por ser muy similares a las acciones y operaciones para el tratamiento de los conceptos, estableciéndose de esta forma el paralelo entre ambos procesos. En el apartado siguiente se presentan las acciones y operaciones de la habilidad profesional [que pretende] diseñar soluciones y visionar estrategias con rigor cientifico.

\section{Acciones y operaciones para la formación inicial de la habilidad profesional. Diseñar soluciones y visionar estrategias con rigor científico con estudiantes de Ingeniería Industrial}

Para el caso de la habilidad profesional que comienza a formarse, se debe lograr que los estudiantes identifiquen las similitudes que hay entre las acciones y operaciones propuestas para el tratamiento de los conceptos, las acciones y operaciones para la formación de la habilidad profesional diseñar soluciones y vislumbrar estrategias con rigor científico. Se reflexiona sobre cada una de las acciones y operaciones, se establecen las semejanzas y diferencias y se les explica que constantemente se estará recurriendo a ellas siempre que se proponga una tarea o problemática, logrando de esta forma alcanzar el diseño y solución de la misma.

Los autores proponen que las acciones y operaciones para la habilidad profesional sean las siguientes:

- Reactivar los medios que tienen implicación con la problemática presentada, recopilando toda la información, independientemente de su utilidad o no. 
En este caso se realizará una clasificación adecuada del problema presentado, para posteriormente determinar qué resoluciones, normas, decretos, etc. tienen relación con la problemática. Se valora la similitud con la acción para el tratamiento de un concepto.

- Determinar un diseño adecuado para la solución del problema planteado.

En esta acción se compara la problemática presentada con otras ya resueltas anteriormente y se valora si se puede realizar un diseño similar, en caso contrario habrá que elaborar un nuevo diseño por tratarse de un problema que no ha sido resuelto con anterioridad.

- Valorar la posibilidad de reducir el problema planteado a otro ya resuelto con anterioridad o cambiar el procedimiento utilizado.

Esta acción está relacionada con la solución propiamente dicha del problema una vez que se ha diseñado dicha solución, se aplicará un procedimiento conocido por tratarse de un problema similar a uno resuelto con anterioridad, en caso contrario entonces habrá que realizar un cambio de procedimiento, repensando así la vía de solución adecuada.

- Presentar ante la organización la estrategia solución (acciones y operaciones previstas para la solución del problema).

Se presenta ante la organización (directivos de la empresa) la estrategia de solución adecuada para el problema, se comentan cuáles son las acciones y operaciones que deben aplicarse para la solución de la problemática presentada.

- Explicar, a los directivos de la organización cada una de las acciones a ejecutarse para la solución del problema propuesto. Se da una explicación detallada de las acciones y operaciones, luego se hacen los comentarios pertinentes para que los 
Domingo Curbeira Hernánder, María de Lourdes Bravo Estévez, Gisela Bravo Lóper.

directivos tengan claridad del porqué de cada una, el objetivo consiste en persuadir y convencer a los directivos de lo acertado de la estrategia de solución que se propone.

- Realizar consideraciones retrospectivas y perspectivas.

Se incorporará en el sistema de procedimientos la medida el diseño de solución propuesto, se analizarán posibles casos límite o extremos según el diseño presentado, se analizará la posibilidad de transferir el diseño presentado a casos similares.

A continuación, desde el punto de vista didáctico, se muestran en una tabla, a modo de resumen parcial, las acciones para ambos procesos donde se ilustra el vínculo o paralelo entre estos:

\begin{tabular}{|l|l|}
\hline \multicolumn{1}{|c|}{$\begin{array}{c}\text { Acciones para el } \\
\text { tratamiento de los conceptos }\end{array}$} & \multicolumn{1}{c|}{$\begin{array}{c}\text { Acciones para la formación } \\
\text { de la habilidad profesional }\end{array}$} \\
\hline $\begin{array}{l}\text { Reactivar los medios que tienen } \\
\text { implicación en el nuevo concepto a } \\
\text { definir, recopilando toda la infor- } \\
\text { mación, independientemente de su } \\
\text { utilidad o no }\end{array}$ & $\begin{array}{l}\text { Reactivar los medios que tienen } \\
\text { implicación con la problemática } \\
\text { presentada, recopilando toda la } \\
\text { información, independientemente } \\
\text { de su utilidad o no }\end{array}$ \\
\hline $\begin{array}{l}\text { Modelar geométricamente el } \\
\text { problema planteado, si es posible }\end{array}$ & $\begin{array}{l}\text { Determinar un diseño adecuado para } \\
\text { la solución del problema planteado }\end{array}$ \\
\hline $\begin{array}{l}\text { Reducir el problema planteado a un } \\
\text { problema resuelto con anterioridad }\end{array}$ & $\begin{array}{l}\text { Valorar la posibilidad de reducir el } \\
\text { problema planteado a otro ya } \\
\text { resuelto con anterioridad o cambiar } \\
\text { el procedimiento utilizado }\end{array}$ \\
\hline $\begin{array}{l}\text { Realizar la construcción de los } \\
\text { objetos correspondientes }\end{array}$ & $\begin{array}{l}\text { Presentar ante la organización la } \\
\text { estrategia de solución (acciones y } \\
\text { operaciones previstas para la solución } \\
\text { del problema) }\end{array}$ \\
\hline $\begin{array}{l}\text { Explicar y/o formular una definición } \\
\text { del concepto sobre la base de los } \\
\text { resultados alcanzados }\end{array}$ & $\begin{array}{l}\text { Explicar, a los directivos de la organi- } \\
\text { zación cada una de las acciones a } \\
\text { aplicar para la solución del problema } \\
\text { propuesto }\end{array}$ \\
\hline $\begin{array}{l}\text { Realizar consideraciones retrospectivas } \\
\text { y perspectivas }\end{array}$ & $\begin{array}{l}\text { Realizar consideraciones retrospectivas } \\
\text { y perspectivas }\end{array}$ \\
\hline
\end{tabular}


La formación inicial de habilidades profesionales del ingeniero industrial desde el contexto de la matemática

\section{Conclusiones}

Del estudio realizado se coligen las siguientes conclusiones:

- La formación de habilidades son asumidas como rasgos pedagógicos y psicológicos, destacándose primero las habilidades generales y luego, como un caso particular, las habilidades profesionales.

- En el proceso de formación de habilidades profesionales se necesita involucrar a los estudiantes como participantes activos del proceso, elaborando con ellos cada una de las acciones y sus correspondientes operaciones o por lo menos proponérselas y debatir sobre cada una de ellas, donde es necesario la motivación, el uso de métodos problémicos [sic] y el tratamiento de situaciones propias de su especialidad.

- El análisis de las concepciones teórico-metodológicas que sustentan el proceso de enseñanza aprendizaje de la Matemática General en el primer año de Ingeniería Industrial permite declarar que este debe concebirse de modo que posibilite la formación, desde lo curricular de las habilidades profesionales que deben ir potenciándose desde este primer año.

- La definición de habilidades profesionales ofrecida tiene como rasgo distintivo que estas se enmarcan desde las diferentes asignaturas del currículo, ya sea de forma implícita o implícita y que son sistematizadas en el proceso de la actividad.

- El establecimiento de un paralelo entre las acciones y operaciones del tratamiento de los conceptos del cálculo integral de la disciplina Matemática General y el proceso de formación inicial de la habilidad profesional "diseñar soluciones y visionar estrategias con rigor cientifico" permite una adecuada contextualización de la enseñanza de la Matemática para el objeto social del Ingeniero Industrial. 
Domingo Curbeira Hernánder, María de Lourdes Bravo Estévez,

Gisela Bravo Lóper.

- La formación desde lo curricular de las habilidades profesionales permite el establecimiento de fuertes relaciones con el entorno y con los paradigmas de la organización al ser considerados problemas propios de la especialidad.

\section{Referencias bibliográficas}

Álvarez de Zayas, R. (1996). El desarrollo de habilidades de la Historia. Honduras: Editorial Guaymuar.

Álvarez de Zayas, C. (1999). La escuela en la vida. La Habana: Pueblo y Educación.

Bermúdez, R., \& Rodríguez, M. (1996). Teoría y metodología del aprendizaje. La Habana: Pueblo y Educación.

Bravo Estévez, M. (2002). Una estrategia didáctica para la enseñanza de las demostraciones geométricas. [Tesis doctoral, Universidad de Oviedo, España].

Brito Fernández, H. (1987). Psicología general para los ISP. La Habana: Pueblo y Educación.

Cañedo Iglesias, C. M. (2004). Estrategia Didáctica para contribuir a la formación de la habilidad profesional esencial "realizar el paso del sistema real al esquema de análisis". En El Ingeniero Mecánico. Tesis en opción al grado científico de doctor en Ciencias Pedagógicas. [Universidad de Cienfuegos "Carlos Rafael Rodríguez”].

Colectivo de autores. (2007). Plan de Estudios D carrera Ingeniería Industrial. La Habana: Comisión Nacional de Carrera.

Dujet, C. (2007). Matemática para Ingenieros. [En línea] Recuperado de http://www.m2real.org/spip.php?article2\&lang=fr. 
La formación inicial de habilidades profesionales del ingeniero industrial desde el contexto de la matemática

Escalona Reyes, M. (2011). El perfeccionamiento de la enseñanza de la Matemática en la Educación Superior. Su concreción en las carreras de ingeniería en la Universidad de Holguín. [En línea]. Recuperado de http:/www.rieoei.org/deloslectores/4410Escalona.pdf.

Fuentes González, H. (1999). Consideraciones sobre la didáctica de la Educación Superior. Serie Educación para Educadores. Santa Fe de Bogotá: UO-CEDINPRO.

Izquierdo Hernández, A. I.; Corona Poveda, A. L. (2012). Didáctica de la educación superior. [En línea]. Recuperado de http://www.monografias.com/trabajo91/didactica-deeducacion-superior/didactica-de-educacion-superior5.shtml.

Márquez Rodríguez, A. (1993). Habilidades. Proposiciones para su evaluación. Santiago de Cuba: Departamento Pedagogía.

Mestre Gómez, U. (1995). La formación profesional en la dinámica del proceso docente-educativo. Revista Cubana de Educación Superior, 2, 20-28.

Miari Casa, A. (1982). Organización y metodología de la enseñanza práctica. La Habana: Pueblo Educación.

Montes de Oca, N. (2002). Una propuesta didáctica para el desarrollo de la habilidad argumentar en el lenguaje de la Matemática en la asignatura Geometría I, en la carrera Licenciatura en Educación, especialidad MatemáticaComputación. [Tesis en opción al grado científico de doctor en Ciencias Pedagógicas, Universidad de Camagüey].

Mulet González, M. A. (2006). Fundamentos psicológicos, pedagógicos y gnoseológicos de las habilidades profesionales pedagógicas. [En línea] Recuperado de http://www.Monografías. com/trabajos37/habilidades-pedagogicas/habilidadespedagogicas.shtml. 
Domingo Curbeira Hernánder, María de Lourdes Bravo Estévez,

Gisela Bravo López.

López Fernández, R. (2010). Componentes para la estructura didáctica de un curso de Educación a Distancia usando como herramienta las plataformas gestoras. [Tesis en opción al grado científico de doctor en Ciencias Pedagógicas, Universidad Pedagógica de Cienfuegos "Conrado Benítez García”].

Llanes Montes, A. (2010). Reflexiones sobre habilidades profesionales de formación específica. [En línea] Recuperado de http://www.monografias.com/trabajos 82/reflexioneshabilidades-profesionales/reflexiones-habilidades-profesionales. shtml.

Ortigoza, C. (2006). Currículum: Diseño, desarrollo y evaluación en la educación superior. Material soporte magnético biblioteca Benito Juárez. Holguín: Universidad de Holguín.

Petrovski, A. V. (1980). Psicología evolutiva y pedagógica. Moscú: Editorial Progreso.

Rodríguez Seijo, I. (2012). Conceptualización y estructura de las habilidades profesionales básicas del técnico de nivel medio en agronomía. [En línea]. Recuperado de http://www.pedagogiaprofesional.rimed.cu.

Talízina, N. (1988). Psicología de la enseñanz̧a. Moscú: Editorial Progreso. 


\section{Domingo Curbeira Hernández}

Es licenciado en Educación, con especialidad de Matemática (1992). Tiene un máster en Ciencias y en Matemática Aplicada (2002). Se ha desempeñado como docente en Educación Superior. Ha impartido varias asignaturas entre las que se destacan: Matemática Básica para Ingeniería Industrial, Álgebra Lineal y Geometría analítica para carreras de Ingeniería, Álgebra I, II, III, IV, V y VI para la formación del profesorado, Matemática I, Matemática II, Matemática III y Matemática IV para la formación de Ingenieros Industriales, Matemática Discreta para la formación de Ingenieros Informáticos. Ha publicado 15 artículos en diversos medios. Ha asesorado tesis de pregrado y tesis de maestría, pero también ha actuado como oponente de varios trabajos de investigación en pregrado y posgrado respectivamente. Actualmente es profesor asistente.

Email: dcurbeira@ucf.edu.cu

\section{María de Lourdes Bravo Estévez}

Es doctora en Ciencias Pedagógicas por la Universidad de Oviedo y tiene un máster en Ciencias de la Educación (2002), licenciada en Educación Especialidad Matemática (1981); profesora de Enseñanza General Media (1979). Durante 33 años se ha desempeñado como profesora de Matemática Superior para las carreras de Ingeniería Mecánica, de Informática e Industrial, de Álgebra Lineal, y de 
Domingo Curbeira Hernánder, María de Lourdes Bravo Estévez, Gisela Bravo Lóper.

Pedagogía en la Universidad de Angola; así como de Geometría Plana y Espacio, Geometría Descriptiva y Geometría Axiomática en la formación de profesores. Profesora de Infotecnología en la maestría de Estudios Sociales, en Venezuela, también ha impartido Matemática Superior en la Maestría de Matemática Aplicada. Participó en el Congreso Nacional de Matemática y Computación y en el VII Simposio de la Sociedad Española de Investigación en Educación Matemática. Sus investigaciones están relacionadas con la enseñanza y aprendizaje de la Geometría y Matemática Superior.

Email: lbravo@ucf.edu.cu

\section{Gisela Bravo López}

Es doctora en Ciencias Pedagógicas por la Universidad de Oviedo, donde obtuvo la categoría de Excelente Cum Lade. Licenciada en Psicología y Pedagogía por la Universidad Pedagógica "Enrique José Varona" (1982). Tiene 23 años de experiencia en la enseñanza de temas psicológicos y pedagógicos. También ha ofrecido numerosos cursos y conferencias sobre comunicación, didáctica, creatividad, manejo de conflicto, Personalidad, Psicología Educativa, métodos de enseñanza, dinámica grupal para profesionales de Brasil, Ecuador, España y Dinamarca. En 1996, obtuvo el grado de máster en Ciencias de la Educación con mención en el tema "La comunicación pedagógica. Un reto al profesor en ejercicio". Durante 1997 ejerció su labor académica en la corporación Universitaria de Ibagué, Colombia, donde impartió numerosos cursos relacionados con las temáticas antes citadas. 
La formación inicial de habilidades profesionales del ingeniero industrial desde el contexto de la matemática

Ha publicado diversos artículos sobre estos temas tanto a nivel nacional como internacional. Actualmente es profesora del Centro de Estudio de la Didáctica y la Dirección de la Educación Superior de la Universidad de Cienfuegos donde imparte varias asignaturas en la maestría sobre Educación.

Email: gbravo@ucf.edu.cu

Recibido: 15/03/2013 Aprobado: 14/06/2013 
